\title{
Pólya PEAKS AND THE OSCILLATION OF POSITIVE FUNCTIONS
}

\author{
DAVID DRASIN ${ }^{1}$ AND DANIEL F. SHEA ${ }^{2}$
}

\begin{abstract}
A new proof is given of the existence of Pólya peaks of an (increasing) function $g(t)$. This approach yields several applications, including a characterization of those $p$ for which $g$ can have peaks of order $p$.
\end{abstract}

Introduction. Many results in function theory depend upon growth properties of increasing functions. For example, the proof of Nevanlinna's second fundamental theorem ultimately depends on some form of a simple but elegant growth lemma due to Borel [11, p. 38]. In recent years, much progress has come from a systematic exploitation of the "Pólya peaks" of an increasing function of finite lower order. that

If $g$ is a positive increasing function on $\left[t_{0}, \infty\right)$, sequences $r_{n}, s_{n} \rightarrow \infty$ such

$$
\begin{array}{ll}
\frac{g(t)}{g\left(r_{n}\right)} \leqq\left(\frac{t}{r_{n}}\right)^{p}\left\{1+\delta_{n}\right\} & \left(a_{n}^{-1} r_{n} \leqq t \leqq a_{n} r_{n}\right), \\
\frac{g(t)}{g\left(s_{n}\right)} \geqq\left(\frac{t}{s_{n}}\right)^{p}\left\{1-\delta_{n}\right\} & \left(a_{n}^{-1} s_{n} \leqq t \leqq a_{n} s_{n}\right)
\end{array}
$$

hold for some $a_{n} \rightarrow \infty, \delta_{n} \rightarrow 0$ are called Pólya peaks of order $p$, of the first and second kinds respectively, for $g$. This definition is due to A. Edrei, who has used these peaks to find a variety of interesting function-theoretic results; see for example [3], [4], [5]. Edrei has shown [4] that peaks of the first kind exist for every $p$ in $\mu \leqq p \leqq \rho$ where

$$
\rho=\limsup _{t \rightarrow \infty} \frac{\log g(t)}{\log t}, \quad \mu=\liminf _{t \rightarrow \infty} \frac{\log g(t)}{\log t}
$$

denote the order and lower order of $g$. When $\rho<\infty$, the existence of peaks of the first kind, order $p=\rho$, can be deduced from a growth lemma of

Received by the editors September 14, 1971 .

AMS 1970 subject classifications. Primary 26A12; Secondary 30A68.

${ }^{1}$ Research supported by NSF grant GP-9454.

${ }^{2}$ Research supported by NSF grant GP-21340. 
Pólya ([11, p. 101], [14]). In [15], Edrei's ideas are adapted to show the existence of peaks of the second kind.

The original methods of Pólya [14] show that, for a convolution transform

$$
G(x)=\int_{0}^{\infty} g(t) k\left(\frac{x}{t}\right) \frac{d t}{t} \quad(0<x<\infty),
$$

with $k$ nonnegative and sufficiently well behaved near 0 and $\infty$,

$$
\liminf _{t \rightarrow \infty} \frac{G(t)}{g(t)} \leqq \hat{k}(\rho) \leqq \limsup _{t \rightarrow \infty} \frac{G(t)}{g(t)}
$$

here $\rho$ is given by (3) and $\hat{k}(\rho)=\int_{0}^{\infty} k(t) t^{-\rho-1} d t$ denotes the Mellin transform of $k$ at $\rho$. Edrei's methods improve (5) by replacing $\rho$ by any number $p$ for which $g$ has Pólya peaks of order $p$ and $\hat{k}(p)<\infty$. Appropriate choices of $g$ and $k$ (cf. [14, pp. 177-180], [8], [4], and item (iii) below) yield interesting value-distribution properties of entire and meromorphic functions.

It is thus of interest to obtain an intrinsic characterization of those $p$ for which Pólya peaks of order $p$ exist, one more manageable than that implied by (1) and (2). Consider

$$
\wp_{1}=\left\{p:(1) \text { holds for some } a_{n} \rightarrow \infty, \delta_{n} \rightarrow 0\right\},
$$

set $\mu_{1}=\inf \left\{p \in \wp_{1}\right\}$ and $\rho_{1}=\sup \left\{p \in \wp_{1}\right\}$, and define $\mu_{2}$ and $\rho_{2}$ similarly in terms of peaks of second kind. Theorem 1 relates these notions to simple oscillation properties of $g$ itself.

THEOREM 1. Let $g$ be positive on $\left[t_{0}, \infty\right)$, and such that

$$
g(x)>\delta g(t)(x / t)^{K} \quad\left(t_{0} \leqq t<x\right)
$$

holds for some $K>-\infty$ and $\delta>0$. Define

$$
\begin{aligned}
& \rho_{*}=\sup \left\{p: \limsup _{x, A \rightarrow \infty} \frac{g(A x)}{A^{p} g(x)}=\infty\right\}, \\
& \mu_{*}=\inf \left\{p: \liminf _{x, A \rightarrow \infty} \frac{g(A x)}{A^{p} g(x)}=0\right\} .
\end{aligned}
$$

Then $g$ has Pólya peaks of first and second kind of order $p(<\infty)$ if and only if

$$
\mu_{*} \leqq p \leqq \rho_{*} .
$$

Thus $\rho_{1}=\rho_{2}=\rho_{*}, \mu_{1}=\mu_{2}=\mu_{*}$. It is obvious directly from (7) and (8) that $\mu$ and $\rho$, defined in (3), satisfy

$$
K \leqq \mu_{*} \leqq \mu \leqq \rho \leqq \rho_{*} \leqq \infty
$$


where $K$ is the constant in (6). Simple examples show that any combination of equality and inequality in (10) is possible.

The weak tauberian condition (6) is equivalent to the one used in [1] and [2]. Although in many applications of Pólya peaks one considers only increasing $g$, our methods apply just as well to $g$ satisfying (6); that some condition like (6) is necessary in Theorem 1 may be seen e.g. from $g(t)=$ $\exp (t \sin t)$.

At the end of this paper, Theorem 1 is applied to give a new proof of a result of Edrei and Fuchs. For the present, we record that the definitions (7), (8) of $\mu_{*}$ and $\rho_{*}$ are relevant in several other contexts:

(i) When $\rho_{*}<\infty$, it follows directly from (6) and (7) that

$$
g(x)<C g(t)(x / t)^{\beta} \quad\left(\rho_{*}<\beta, C=C(\beta) ; t_{0} \leqq t<x\right) .
$$

Inequalities like (11) are often needed in tauberian problems to estimate the "tails" of convolutions (4); see for instance [2, $\S 4$ and 5] where these ideas are decisive, and $[9, \S 8]$.

(ii) When $g$ is positive, measurable and regularly varying (in Karamata's sense) so that for each fixed $A>0 \lim _{x \rightarrow \infty} g(A x) / g(x)=A^{\rho}$ for some $\rho$, the estimate

$$
A^{\rho-\varepsilon}<g(A x) / g(x)<A^{\rho+\varepsilon} \quad\left(\varepsilon>0 ; x \geqq x_{0}, A \geqq A_{0}\right)
$$

is classical and useful ([9], [13]). A glance at our definitions (7) and (8) shows that the class of positive functions with $\mu_{*}=\rho_{*}=\rho$ is precisely the class for which (12) holds.

(iii) Finally, we give a typical application to function theory (compare Theorem 1 of [5]). Let $f(z)$ be an entire function normalized by $f(0)=1$, and let $M(t, f)=\max _{\theta}\left|f\left(t e^{i \theta}\right)\right|, L(t, f)=\min _{\theta}\left|f\left(t e^{i \theta}\right)\right|$. The method of Denjoy-Kjellberg [12] shows (implicitly on pp. 193-196 of [12]) that if $0<\alpha<1$, there exist positive constants $k(\alpha)$ and $C(\alpha)$ with

$$
\begin{aligned}
\int_{r}^{R}\{\log L(t, f)-\cos \pi \alpha \log M(t, f)\} \frac{d t}{t^{1+\alpha}} \\
\\
\geqq k(\alpha) \frac{\log M(r, f)}{r^{\alpha}}-C(\alpha) \frac{\log M(4 R, f)}{R^{\alpha}}
\end{aligned}
$$

when $0<r<R$. Thus, if $\mu_{*}$ [defined in (8) with $g(t)=\log M(t, f)$ ] is less than one, the left side of (13) is positive for $\alpha \in\left(\mu_{*}, 1\right)$ for arbitrarily large $r, R / r$ and so

$$
\limsup _{t \rightarrow \infty} \frac{\log L(t, f)}{\log M(t, f)} \geqq \cos \pi \mu_{*} .
$$


This is a sharpened form of the classical "cos $\pi \rho$ theorem" which does not involve the order or lower order of $f$.

1. One-sided peaks. Our existence theorem for Pólya peaks will be a simple consequence of

THEOREM 1a. Let $g$ be positive and continuous on $\left[t_{0}, \infty\right)$, and such that (6) holds there. Let $x_{n}, A_{n} \rightarrow \infty$ be given, and define $p_{n}$ by

$$
g\left(A_{n} x_{n}\right) / g\left(x_{n}\right)=A_{n}^{p_{n}} \quad(n \geqq 1) .
$$

Then there exist sequences $r_{n}, r_{n}^{\prime}, s_{n}, s_{n}^{\prime}, a_{n}$ all tending to $\infty$ and $\varepsilon_{n}, \delta_{n} \downarrow 0$ such that

$$
\begin{array}{ll}
\frac{g(t)}{g\left(r_{n}\right)} \leqq\left\{1+\delta_{n}\right\}\left(\frac{t}{r_{n}}\right)^{p_{n}\left(1-\varepsilon_{n}\right)} & \left(a_{n}^{-1} r_{n} \leqq t \leqq r_{n}\right), \\
\frac{g(t)}{g\left(r_{n}^{\prime}\right)} \leqq\left\{1+\delta_{n}\right\}\left(\frac{t}{r_{n}^{\prime}}\right)^{p_{n}\left(1+\varepsilon_{n}\right)} & \left(r_{n}^{\prime} \leqq t \leqq a_{n} r_{n}^{\prime}\right), \\
\frac{g(t)}{g\left(s_{n}\right)} \geqq\left\{1-\delta_{n}\right\}\left(\frac{t}{s_{n}}\right)^{p_{n}\left(1+\varepsilon_{n}\right)} & \left(a_{n}^{-1} s_{n} \leqq t \leqq s_{n}\right), \\
\frac{g(t)}{g\left(s_{n}^{\prime}\right)} \geqq\left\{1-\delta_{n}\right\}\left(\frac{t}{s_{n}^{\prime}}\right)^{p_{n}\left(1-\varepsilon_{n}\right)} & \left(s_{n}^{\prime} \leqq t \leqq a_{n} s_{n}^{\prime}\right) .
\end{array}
$$

Proof. Assume first that (6) holds with

$$
K=1
$$

so that

$$
\liminf _{n \rightarrow \infty} p_{n} \geqq 1 .
$$

Choose $\varepsilon_{n}<1, \varepsilon_{n} \downarrow 0$ so slowly that $A_{n}^{\varepsilon_{n}} \rightarrow \infty$. Define

$$
r_{n}=\inf \left\{t \in\left[x_{n}, A_{n} x_{n}\right]: \frac{g(t)}{g\left(A_{n} x_{n}\right)} \geqq\left(\frac{t}{A_{n} x_{n}}\right)^{p_{n}\left(1-\varepsilon_{n}\right)}\right\} ;
$$

by (1.1), $r_{n}>x_{n}$. The point of this choice of $r_{n}$ is explained by the following estimate: for $x_{n} \leqq t \leqq r_{n}$,

$$
\begin{aligned}
\frac{g(t)}{g\left(r_{n}\right)} & =\frac{g(t)}{g\left(A_{n} x_{n}\right)}\left(\frac{A_{n} x_{n}}{r_{n}}\right)^{p_{n}\left(1-\varepsilon_{n}\right)} \\
& \leqq\left(\frac{t}{A_{n} x_{n}}\right)^{p_{n}\left(1-\varepsilon_{n}\right)}\left(\frac{A_{n} x_{n}}{r_{n}}\right)^{p_{n}\left(1-\varepsilon_{n}\right)}=\left(\frac{t}{r_{n}}\right)^{p_{n}\left(1-\varepsilon_{n}\right)} .
\end{aligned}
$$


Thus if $r_{n} / x_{n} \rightarrow \infty$, we can set $a_{n}=r_{n} / x_{n}$ and (1.2) follows. When $\lim \inf \left\{r_{n} \mid x_{n}\right\}<\infty$, we deduce from (1.8) and (1.1) that

$$
\begin{aligned}
\frac{g\left(r_{n}\right)}{g\left(x_{n}\right)} & =\frac{g\left(A_{n} x_{n}\right)}{g\left(x_{n}\right)} \frac{g\left(r_{n}\right)}{g\left(A_{n} x_{n}\right)} \\
& =A_{n}^{p_{n}}\left(\frac{r_{n}}{A_{n} x_{n}}\right)^{p_{n}\left(1-\varepsilon_{n}\right)} \\
& =A_{n}^{\varepsilon_{n} p_{n}}\left(\frac{r_{n}}{x_{n}}\right)^{p_{n}\left(1-\varepsilon_{n}\right)}
\end{aligned}
$$

and thus

$$
g\left(r_{n}\right) / g\left(x_{n}\right)>A_{n}^{\varepsilon_{n} p_{n}} .
$$

Now choose $y_{n}\left(<x_{n}\right.$ for all large $n$, by (1.7)) so that

$$
\left(r_{n} / y_{n}\right)^{p_{n}\left(1-\varepsilon_{n}\right)}=g\left(r_{n}\right) / g\left(x_{n}\right) .
$$

Thus (1.11) and (1.7) imply

$$
r_{n} / y_{n}>A_{n}^{e_{n}} \rightarrow \infty
$$

and by (6), (1.6) and (1.12)

$$
\frac{g(t)}{g\left(r_{n}\right)}<\frac{g\left(x_{n}\right)}{\delta g\left(r_{n}\right)}=\frac{1}{\delta}\left(\frac{y_{n}}{r_{n}}\right)^{p_{n}\left(1-\varepsilon_{n}\right)} \quad\left(y_{n} \leqq t \leqq x_{n}\right) .
$$

Putting $z_{n}=\min \left(x_{n}, \delta^{-1 / p_{n}\left(1-\varepsilon_{n}\right)} y_{n}\right)$, we have from (1.13) and (1.7) that $r_{n} / z_{n} \rightarrow \infty(n \rightarrow \infty)$, and from (1.14) and (1.9) that

$$
g(t) / g\left(r_{n}\right) \leqq\left(t / r_{n}\right)^{p_{n}\left(1-\varepsilon_{n}\right)} \quad\left(z_{n} \leqq t \leqq r_{n}\right) .
$$

This proves (1.2), with $a_{n}=r_{n} / z_{n}$ and $\delta_{n}=0$.

The proof of (1.3) is easier; define

$$
r_{n}^{\prime}=\sup \left\{t \in\left[x_{n}, A_{n} x_{n}\right]: \frac{g(t)}{g\left(x_{n}\right)} \geqq\left(\frac{t}{x_{n}}\right)^{p_{n}\left(1+\varepsilon_{n}\right)}\right\} .
$$

Then we have from (6), (1.6), (1.1) and (1.15) that

$$
\delta<\frac{g\left(A_{n} x_{n}\right)}{g\left(r_{n}^{\prime}\right)}=A_{n}^{p_{n}}\left(\frac{x_{n}}{r_{n}^{\prime}}\right)^{p_{n}\left(1+\varepsilon_{n}\right)}=\left(\frac{A_{n} x_{n}}{r_{n}^{\prime}}\right)^{p_{n}\left(1+\varepsilon_{n}\right)} A_{n}^{-\varepsilon_{n} p_{n}},
$$

so that by (1.7),

$$
A_{n} x_{n} / r_{n}^{\prime} \geqq \eta A_{n}^{\varepsilon_{n} /\left(1+\varepsilon_{n}\right)} \rightarrow \infty \quad(n \rightarrow \infty)
$$


for some $\eta>0$. Also, on $r_{n}^{\prime} \leqq t \leqq A_{n} x_{n}$ we have

$$
g(t) / g\left(r_{n}^{\prime}\right)=g(t) / g\left(x_{n}\right)\left(x_{n} / r_{n}^{\prime}\right)^{p_{n}\left(1+\varepsilon_{n}\right)} \leqq\left(t / r_{n}^{\prime}\right)^{p_{n}\left(1+\varepsilon_{n}\right)},
$$

and thus (1.3) is a consequence of (1.16) and (1.17).

To verify (1.5) one defines, analogously to (1.8),

$$
s_{n}^{\prime}=\sup \left\{t \in\left[x_{n}, A_{n} x_{n}\right]: \frac{g(t)}{g\left(x_{n}\right)} \leqq\left(\frac{t}{x_{n}}\right)^{p_{n}\left(1-\varepsilon_{n}\right)}\right\},
$$

and proceeds as in the proof of (1.2). Finally, using

$$
s_{n}=\inf \left\{t \in\left[x_{n}, A_{n} x_{n}\right]: \frac{g(t)}{g\left(A_{n} x_{n}\right)} \leqq\left(\frac{t}{A_{n} x_{n}}\right)^{p_{n}\left(1+\varepsilon_{n}\right)}\right\},
$$

(1.4) can be obtained in the same manner as was (1.3).

When $g$ fails to satisfy (1.6), define $h(t)=g(t) t^{1-K}$. Then the hypotheses of Theorem la as well as (1.6) are satisfied by $h$, and the above proof yields (1.2)-(1.5) with $g$ replaced by $h, p_{n}$ replaced by $q_{n} \equiv p_{n}+1-K$, and $\delta_{n} \equiv 0$. Decreasing the $a_{n}$ if necessary to achieve $a_{n}^{\varepsilon_{n}}=1+o(1)(n \rightarrow \infty)$, the conclusion of Theorem la follows easily.

2. Proof of Theorem 1. From (1), (2) we find that there exist $A_{n}, B_{n}$, $x_{n}, y_{n} \rightarrow \infty$ such that

$$
\frac{g\left(A_{n} x_{n}\right)}{A_{n}^{p} g\left(x_{n}\right)} \leqq 1+o(1) \leqq \frac{g\left(B_{n} y_{n}\right)}{B_{n}^{p} g\left(y_{n}\right)} \quad(n \rightarrow \infty)
$$

(Consider $r_{n}, a_{n}, a_{n}^{-1} r_{n}$ if (1) holds, for instance.) Hence (9) is necessary for (1) and (2).

For sufficiency, let $p(<\infty)$ satisfy (9), and assume first that $g$ is continuous. Choose $A_{n}, x_{n} \rightarrow \infty$ so that the $p_{n}$ in (1.1) satisfy $p_{n} \rightarrow \rho_{*}(n \rightarrow \infty)$, and choose $B_{n}, y_{n} \rightarrow \infty$ so that the $q_{n}$ defined by $g\left(B_{n} y_{n}\right) / g\left(y_{n}\right)=B_{n}^{a_{n}}$ satisfy $q_{n} \rightarrow \mu_{*}(n \rightarrow \infty)$. Then there exist $\eta_{n} \downarrow 0$ such that

$$
p-\eta_{n}<p_{n}, \quad q_{n}<p+\eta_{n} \quad(n \geqq 1) .
$$

Using the first inequality of (2.1) in (1.2), and decreasing the $a_{n}$ if necessary to achieve $a_{n}^{\eta_{n}+\varepsilon_{n} \rightarrow 1}$, we find $r_{n}^{*} \rightarrow \infty$ such that

$$
\begin{aligned}
\frac{g(t)}{g\left(r_{n}^{*}\right)} & \leqq\left\{1+\delta_{n}\right\}\left(\frac{t}{r_{n}^{*}}\right)^{\left(p-\eta_{n}\right)\left(1-\varepsilon_{n}\right)} \\
& =\left(\frac{t}{r_{n}^{*}}\right)^{p}\{1+o(1)\} \quad\left(a_{n}^{-1} r_{n}^{*} \leqq t \leqq r_{n}^{*}\right)
\end{aligned}
$$


The second part of (2.1) used in (1.3) yields $r_{n}^{\prime} \rightarrow \infty$ satisfying

$$
\begin{aligned}
\frac{g(t)}{g\left(r_{n}^{\prime}\right)} & \leqq\left\{1+\delta_{n}\right\}\left(\frac{t}{r_{n}^{\prime}}\right)^{\left(p+\eta_{n}\right)\left(1+\varepsilon_{n}\right)} \\
& =\left(\frac{t}{r_{n}^{\prime}}\right)^{p}\{1+o(1)\} \quad\left(r_{n}^{\prime} \leqq t \leqq a_{n} r_{n}^{\prime}\right)
\end{aligned}
$$

By passing to subsequences if necessary we may assume that $r_{n}^{*}<r_{n}^{\prime}$, as well as (2.2) and (2.3), holds for all $n \geqq 1$. Choosing $r_{n} \in\left[r_{n}^{*}, r_{n}^{\prime}\right]$ by

$$
\frac{g\left(r_{n}\right)}{r_{n}^{p}}=\sup _{r_{n}^{*} \leqq t \leqq r_{n}^{\prime}} \frac{g(t)}{t^{p}}
$$

we have (1) when $g$ is continuous. For the general case, put $t_{n}=t_{0}+n$ and consider the step function

$$
\begin{aligned}
& g_{0}(x)=\sup _{t_{n} \leqq t \leqq t_{n+1}} g(t) \quad\left(t_{n}<x<t_{n+1}\right), \\
& g_{0}\left(t_{n}\right)=\sup \left\{g_{0}\left(t_{n}+\right), g_{0}\left(t_{n}-\right)\right\} \quad(n \geqq 1) .
\end{aligned}
$$

It is not difficult to approximate $g_{0}$ by a continuous function $g_{1} \geqq g_{0}$ satisfying (6) and such that the values of $\rho_{*}, \mu_{*}$ in (7), (8) are not altered when $g$ is replaced by $g_{1}$. Further, if $r_{n} \rightarrow \infty$ satisfies (1) with $g_{1}$ in place of $g$, then there exist $R_{n} \in\left[r_{n}-1, r_{n}+1\right]$ such that $g\left(R_{n}\right) \sim g_{1}\left(R_{n}\right) \sim g_{1}\left(r_{n}\right)$ $(n \rightarrow \infty)$, and thus $g$ satisfies (1) with $r_{n}$ replaced by $R_{n}$.

The proof of (2) uses (1.4) and (1.5) in an analogous manner.

3. An application. Theorem 1 allows a new interpretation, as well as a short proof, of a result of Edrei and Fuchs ([6, Theorem 5], [7, Lemma A]). Let $f(z)$ be entire, of lower order $\mu<\infty$ and order $\rho(\leqq \infty)$ and put

$$
\mathscr{K}=\mathscr{K}(f)=\limsup (N(r, 0) / T(r, f)) .
$$

Edrei and Fuchs have shown ([3, Theorem 4a] [10, Lemma 9.2]) that, for any integer $q \geqq 0$,

$$
\begin{aligned}
(2-\mathscr{K}-\varepsilon) g(r) & <(\mathscr{K}+\varepsilon) A(q+1) \int_{s}^{R} g(t)\left(\frac{r}{t}\right)^{q} k\left(\frac{r}{t}\right) \frac{d t}{t} \\
+ & B\left(\frac{s}{r}\right)^{q} g(2 s)+B\left(\frac{r}{R}\right)^{a+1} g(2 R) \quad\left(s_{0}(\varepsilon) \leqq s \leqq \frac{1}{2} r \leqq \frac{1}{4} R\right)
\end{aligned}
$$

where $g(t)=T(t, f), \varepsilon>0$ and $A, B$ denote absolute constants. Using (1) and an estimate [6, p. 302] for $\hat{k}(p-q)$, we obtain

$$
\mathscr{K}(f) \geqq \eta \sup _{\mu_{*} \leqq p \leqq \rho_{*}} \frac{|\sin \pi p|}{p} \quad(\eta=\text { absolute constant }) .
$$


Thus if $\mathscr{K}(f)<\delta$ for small enough $\delta$, the fact that peaks exist throughout the entire interval $\left[\mu_{*}, \rho_{*}\right]$ yields

$$
\left|\mu_{*}-n\right|<\frac{1}{4}, \quad \rho_{*}-\mu_{*}<\frac{1}{4},
$$

say, for some positive integer $n$.

Since $g$ does not have Pólya peaks of order $n+\frac{1}{2}$, Theorem 1 implies that there exist $x_{0}, A$ with $g(u) u^{-n-1 / 2} \leqq g(x) x^{-n-1 / 2}$ for all $u$ and $x$ which satisfy $x_{0}<x \leqq u \mid A$. For any $x>x_{0}$, choose $t \in[x, A x]$ with $g(t) t^{-n-1 / 2}=$ $\sup _{x \leqq u \leqq A x} g(u) u^{-n-1 / 2}$, and it is then clear that each interval $[x, A x]$ has a point $t$ with

$$
g(u) u^{-n-1 / 2} \leqq g(t) t^{-n-1 / 2} \quad(t \leqq u<\infty) .
$$

This is essentially part II of Theorem 5 in [6], and part I may be obtained similarly.

ADDED IN PROOF. In a recent paper (Properties of Pólya peaks, Rocky Mt. J. Math. 1 (1971), 649-656), H. Silverman states a necessary condition for the existence of Pólya peaks of order $p$, namely: $\kappa \leqq p \leqq \Omega$, where $\kappa$ and $\Omega$ are obtained as certain iterated limits (loc. cit., p. 651). It is not difficult to see that in fact $\kappa=\mu_{*}$ and $\Omega=\rho_{*}$; thus, by Theorem 1 above, Silverman's condition is sufficient as well as necessary.

\section{REFERENCES}

1. D. Drasin, Tauberian theorems and slowly varying functions, Trans. Amer. Math. Soc. 133 (1968), 333-356. MR 37 \#1607.

2. D. Drasin and D. F. Shea, Convolution inequalities, regular variation and exceptional sets (to appear).

3. A. Edrei, The deficiencies of meromor phic functions of finite lower order, Duke Math. J. 31 (1964), 1-21. MR 28 \#1306.

4. —_, Sums of deficiencies of meromorphic functions, J. Analyse Math. 14 (1965), 79-107. MR 31 \#4909.

5. - A local form of the Phragmén-Lindelöf indicator, Mathematika 17 (1970), 149-172. MR 42 \#2000.

6. A. Edrei and W. H. J. Fuchs, On the growth of meromorphic functions with several deficient values, Trans. Amer. Math. Soc. 93 (1959), 292-328. MR 22 \#770.

7. - - Valeurs déficientes et valeurs asymptotiques des fonctions méromorphes, Comment. Math. Helv. 33 (1959), 258-295. MR 23 \#A1038.

8. - The deficiencies of meromorphic functions of order less than one, Duke Math. J. 27 (1960), 233-249. MR 23 \#A1039.

9. W. Feller, On regular variation and local limit theorems, Proc. Fifth Berkeley Sympos. Math. Statist. and Probability (Berkeley, Calif., 1965/66), vol. II: Contributions to Probability Theory, part I, Univ. of California Press, Berkeley, Calif., 1967, pp. 373-388. MR 36 \#2200.

10. W. H. J. Fuchs, Théorie de l'approximation des fonctions d'une variable complexe, Séminaire de Mathématiques Supérieures, no. 26 (Été 1967), Les Presses de l'Université de Montréal, Que., 1968. MR 41 \#5630. 
11. W. K. Hayman, Meromorphic functions, Oxford Math. Monographs, Clarendon Press, Oxford, 1964. MR 29 \#7337.

12. B. Kjellberg, On the minimum modulus of entire functions of lower order less than one, Math. Scand. 8 (1960), 189-197. MR 23 \#A3264.

13. J. Korevaar, T. van Aardenne-Ehrenfest and N. G. de Bruijn, A note on slowly oscillating functions, Nieuw Arch. Wisk. (2) 23 (1949), 77-86. MR 10, 358.

14. G. Pólya, Bemerkungen über unendliche Folgen und ganze Funktionen, Math. Ann. 88 (1923), 169-83.

15. D. F. Shea, On the Valiron deficiencies of meromorphic functions of finite order, Trans. Amer. Math. Soc. 124 (1966), 210-227. MR 34 \#351.

Department of Mathematics, Purdue University, West Lafayette, Indiana 47907

Department of Mathematics, University of Wisconsin, Madison, Wisconsin 53706 